\title{
Relationship between the technical condition of the equipment of heat and power engineering units and the noise level in the environment
}

\author{
O A Gorbunova*, G I Pavlov, $P$ V Nakoryakov and $Y u$ I Khakimzyanova \\ Kazan National Research Technical University named after A.N. Tupolev, 10 Karl Marks street, Kazan, Russian Federation
}

\begin{abstract}
The problem of environmental noise pollution from large-city centralized heating systems is considered. Based on the analysis of acoustic characteristics and vibration parameters of district boiler house power plants, the main reasons for the generation of increased noise are identified. The relationship between the technical condition of the power equipment, buildings, structures and the noise level in the environment is revealed. The possibility of developing complexes of technical measures to reduce noise pollution adjacent to the district boiler houses of the city is shown. While optimizing the selected variants of noise and vibration dampening complexes, it seems promising to use a calculated model of the noise field.
\end{abstract}

\section{Introduction}

Urban (district) boiler houses are included in the general energy system of large cities. They are designed to provide heating and hot water to the city's vast residential areas and industrial complexes. Boiler houses are a system of thermal power plants located on special isolated premises.

It is known that heat and power facilities have a negative impact on the environment: air pollution with harmful gas emissions, the formation of sludge, slag, wastewater, etc. In addition, the operation of boilers, thermal power plants, and thermoelectric power plants is accompanied by the formation of noise. The round-theclock activity of heat and power enterprises negatively impacts the environment both day and night. District boiler houses are often located near residential areas. The population in these areas is subject to noise levels in significant excess of the established standards. The main complaints are: poor health, headaches, sleep disturbances, and impaired cardiovascular function. Due to the development of the territory adjacent to heat power enterprises, residential buildings or the expansion of production capacities of the enterprises themselves, the established sanitary protection zones do not meet the requirements [1-3]. The wear and tear of the equipment and other factors associated with troubleshooting of equipment also contribute to noise levels.

\section{Object and methods}

The centralized heating system of the city of Kazan includes three district boiler houses $(\mathrm{BH})$ with thermal capacities of $200 \mathrm{Gcal} /$ hour (BH Gorki), $360 \mathrm{Gcal} /$ hour (BH Azino) and $540 \mathrm{Gcal} /$ hour (BH Savinovo).
As a result of intensive residential, urban boiler houses were located inside dense urban areas. The levels of total noise near enterprises at night during the heating period are 68-71 dBA (BH Savinovo), 58-62 dBA (BH Gorki), 61-65 dBA (BH Azino). These noise levels significantly exceed sanitary code.

To develop noise protection measures, first of all, it is necessary to identify the noise sources, and to determine the reasons for increased noise levels. For this purpose, the acoustic characteristics of the power equipment were investigated. The condition and serviceability of the attachment points and mounting frames of the boiler house power equipment were evaluated by examining and measuring their vibration parameters. The research results identified the main reasons for the increased noise levels: most of the power plants are morally and physically outdated; the walls of the workshops do not have soundproof coatings, there are acoustic leaks in the structural elements of buildings, there is no glazing in a number of windows; the foundations of power plants are not acoustically decoupled from a common foundation; pipelines are not equipped with vibration compensators, etc. The identified reasons are systemic in nature, i.e. they are characteristic of almost all investigated power system components.

The revealed deviations are most likely associated with the long-term operation of thermal power facilities (boiler houses were commissioned in the late 1980s), repair work in disturbances of the technological regulations, the appearance of defects on the envelope of buildings and structures due to natural aging, loosening mounting frames, etc. These disturbances contribute to an increase in equipment vibration, the appearance of structural noise, and the propagation of large-amplitude noise over significant distances both in the structure of

Corresponding author: rox-22@mail.ru 
buildings and in the air. This is confirmed by experimental results - an analysis of the amplitudefrequency characteristics of vibration and acoustic signals recorded for identified sources.

\section{Results}

Figures 1-4 show the spectra of vibration and acoustic signals of a water pump, a pipeline, as well as a section of the wall where the pipeline is supported. Signals were recorded simultaneously.

The pump park of the BH Gorki includes 4 centrifugal pumps SE-800-100 and one "summer" pump D630-90. With a typical circuit of boiler, 2-3 pumps are in operation [4]. During the inspection of the premises, it was noted that the main pumps and motors are mounted on concrete bases without vibration damping substrates or supports, and there are no vibration isolating gaskets at the support points of water pipes.

The highest sound pressure levels in the amplitudefrequency spectrum of the SE-800-100 engine (Fig. 1 b) are observed in the low frequency octave bands, in the medium frequency range $(600-750 \mathrm{~Hz})$ and in the high frequency range $(1100 \mathrm{~Hz}, 1600-1750 \mathrm{~Hz}, 2500-2600$ $\mathrm{Hz}, 3500 \mathrm{~Hz}, 4600 \mathrm{~Hz}$ ). The reason for their occurrence is the operation of the electric motor (Fig. 1b). The electric motor is connected to the pump by a rigid connection. Therefore, vibration oscillations occurring in

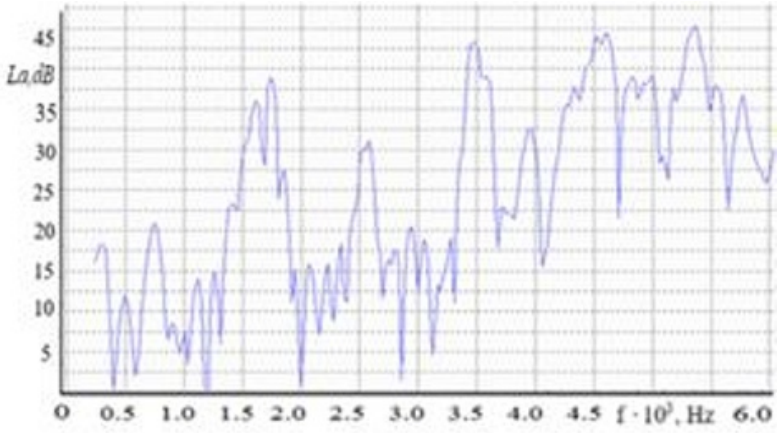

a)

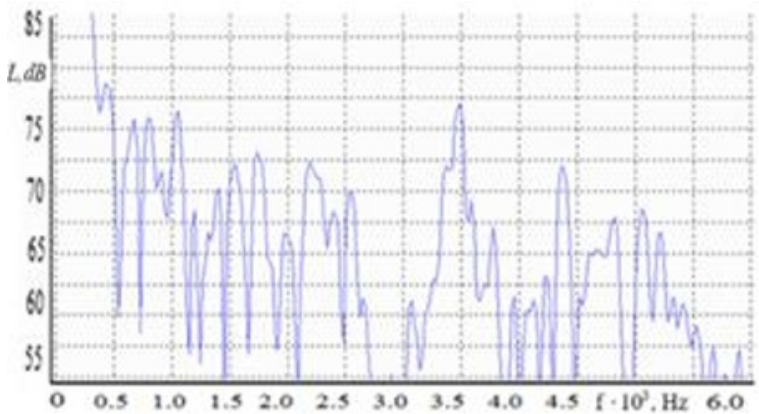

b)

Fig. 1. Spectra of vibration (a) and acoustic (b) motor signals (315 kW, $1450 \mathrm{rpm})$ of a network pump SE-800-100 (BH Gorki).

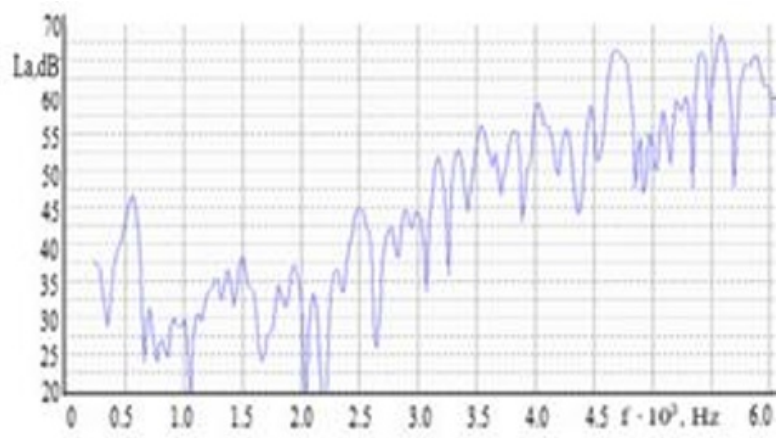

a)

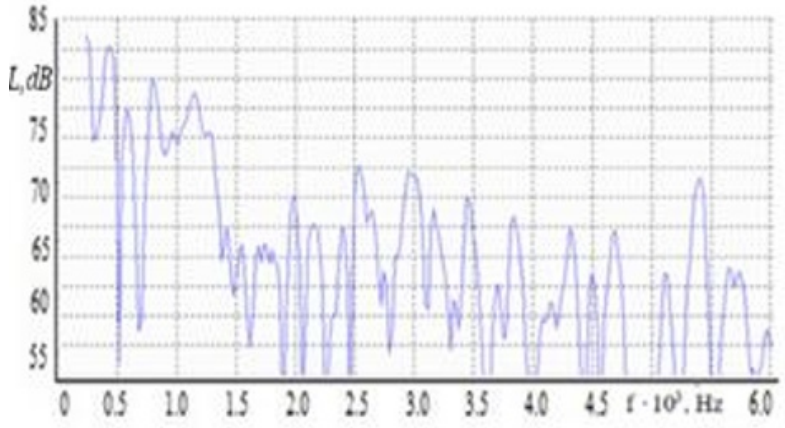

b)

Fig. 2. Spectra of vibration (a) and acoustic (b) signals of the network pump SE-800-100 (BH Gorki).

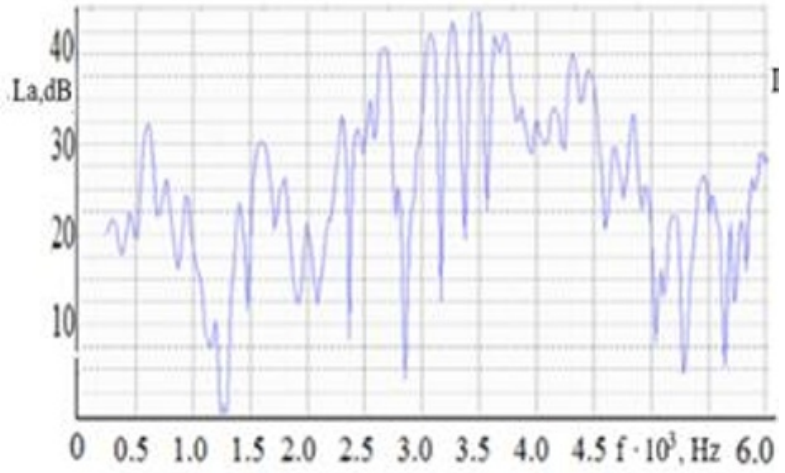

a)

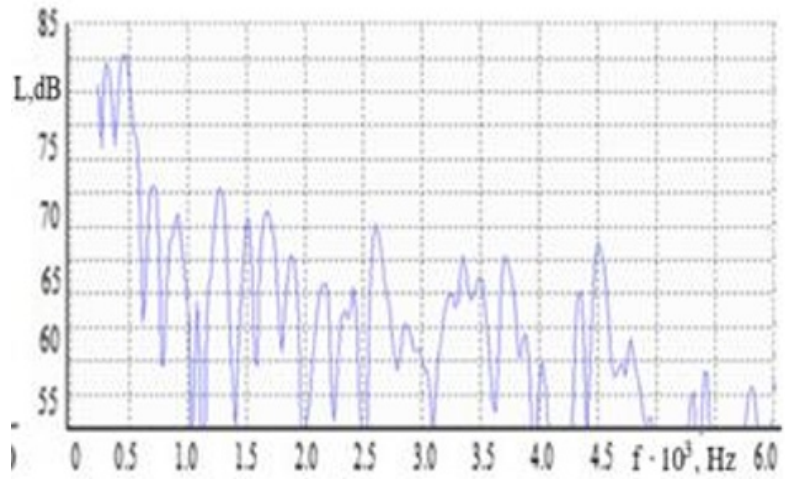

b) 


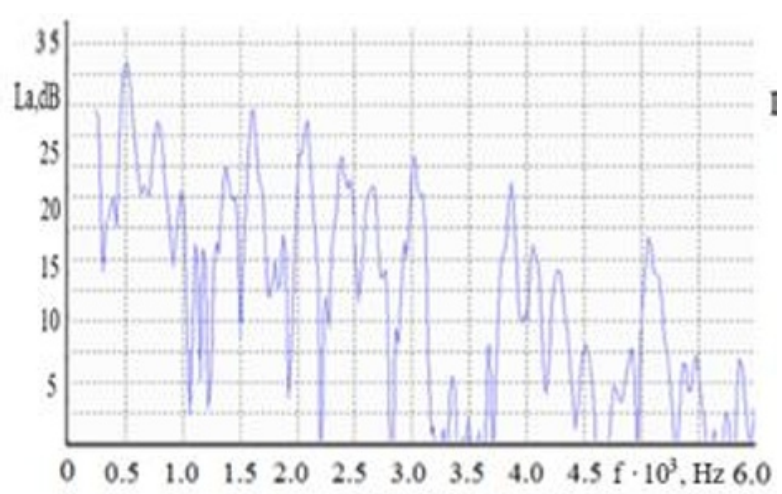

a)

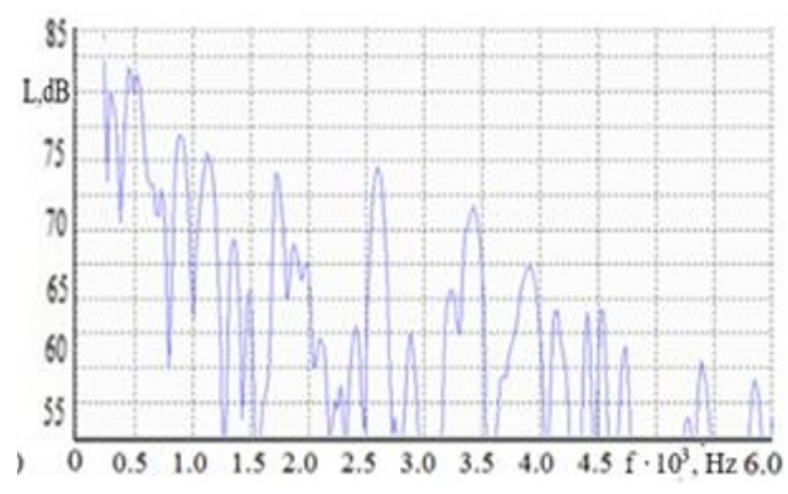

b)

Fig. 4. Spectra of vibration (a) and acoustic (b) signals (vibration sensor is mounted on the wall of the BH Gorki).

the electric motor are freely transmitted to the water pump. The spectral characteristic of the SE-800-100 pump (Fig. 2 a) vibratory signal contains "energycarrying frequencies" in the low-frequency range and in the high-frequency range of 1500-1600 Hz, 2500-2600 $\mathrm{Hz}, 3500 \mathrm{~Hz}, 4600 \mathrm{~Hz}$, which were also observed in the vibration spectrum of the electric motor. Oscillations in the pump casing generate structural noise that spreads in the surrounding area. Vibrations from the pump casing are also transmitted through the pipeline ("energycarrying frequencies": $600 \mathrm{~Hz}, 1600 \mathrm{~Hz}, 2600 \mathrm{~Hz}, 3000$ $3500 \mathrm{~Hz}$ (Fig. $3 \mathrm{a}$ )) and then to the supporting structures of the boiler building. On the spectra of the vibration signal there are frequencies that are present in the respective frequency spectra of the pump, the electric motor supplying it and the pipeline of the network water.

The conducted studies allowed us to establish the relationships between the noise level in the environment and the technical condition of auxiliary equipment, buildings and structures of the $\mathrm{BH}$ Gorki, BH Savinovo [5] and BH Azino. Further, based on these results, a search and selection of methods and means of reducing noise and vibration was carried out in relation to each specific heat supply facility.

There are many means and methods of dealing with noise and vibrations [6-13]. Each means of noise and vibration dampening, individually or in combination with others, can provide noise reduction to the required level. An important task is to choose the best option. At the same time, the economic criterion is taken as the main optimization criterion, that is, from all possible variants of the noise and vibration dampening complexes, the one that is least costly is selected. Carrying out manual calculations to assess the

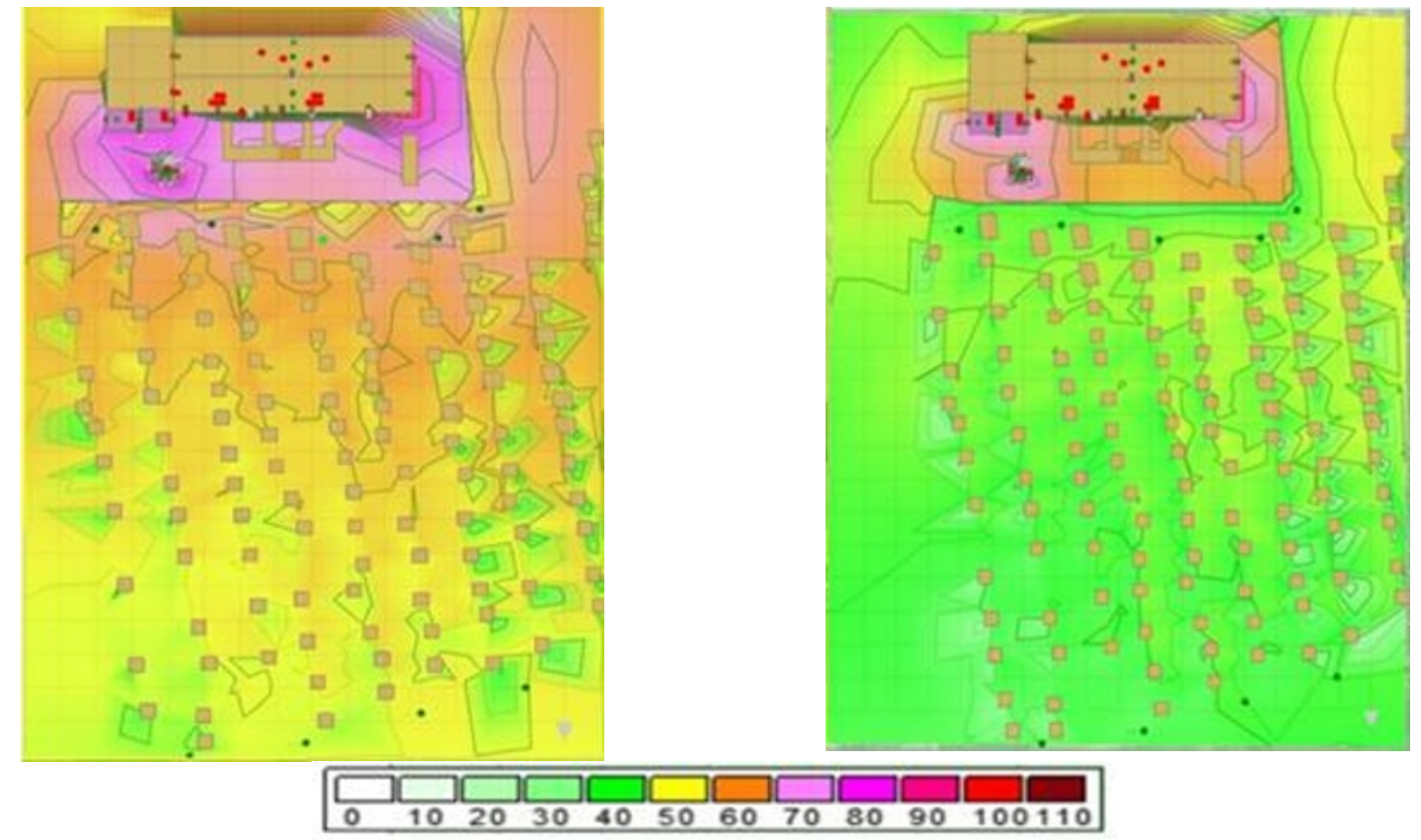

a)

b)

Fig. 5. Noise cards of the sanitary protection zone of the BH Savinovo: a) prior to the implementation of the proposed set of activities; b) after the implementation of the proposed set of activities. 
effectiveness of various options for noise and vibration dampening complexes is very time consuming. The use of the calculation model seems promising. To build such models, it is advisable to use dedicated calculation programs that take into account geographical features and the influence of various factors. The main software systems include: SoundPLAN (Germany), Predictor (Germany), ARM Acoustics (Russia), Ecolog-Shoom (Russia), Garant-shoom (Russia). An example of using the ARM Acoustics software package [14] is presented in [15]. Figure 5 shows the results of computational studies presented in the form of a noise field: a - the current situation, $b$ - the calculated acoustic situation after the introduction of the noise and vibration dampening complex. The noise in the study area was created by the power plants of the Savinovo boiler house.

The noise field calculation model allows optimization of various options for combining means and methods for reducing noise and vibration and choosing the most economically viable option.

\section{Conclusion}

The main reasons for the reduction of the sanitary protection zone of district boiler houses includes the construction of residential buildings in nearby areas, as well as the moral and physical obsolescence of auxiliary power equipment. The identified causes are systemic.

The specific reasons for the increased noise generation of the identified noise sources can be established on the basis of the analysis of acoustic and vibration signals recorded simultaneously and assessment of the technical condition of the mounting points and equipment installation.

Determining the relationship between noise level in the environment and the technical condition of auxiliary equipment, buildings and structures used in the heat supply system allow the development of technical measures to improve the acoustic situation in the areas adjacent to the district boiler houses.

While optimizing the selected variants of noise and vibration protective measures, the use of a calculation model seems promising.

\section{References}

1. SN 2.2.4 / 2.1.8.562-96 Noise at workplaces, in residential, public buildings and in residential areas.

2. V.B. Tupov, Factors of Physical Impact of Thermal Power Plants on the Environment, 284 (2012)

3. A. Yakimovich, A. Vasilyev, V. Vasilyev, Methods and Results of Monitoring of Acoustic Pollution of Urbanized Territories on the Example of the Samara Region of Russia, Ecology and Industry of the Russian Federation 23(6), 28-33 (2019) https://doi.org/10.18412/1816-0395-2019-6-28-33

4. T.I. Safuanov, Improving the Energy Efficiency of the Boiler House "Gorki", Vestnik of Kazan State Power Engineering University, 3(27) (2015)
5. O.A. Gorbunova, G.I. Pavlov, P.V. Nakoryakov, Development of Design Solutions to Reduce Noise from the Boiler House to Protect the Public, Ecology and Industry of Russia, 21(10), 44-49 (2017)

6. V.B. Tupov, Development of methods for reducing noise pollution of the environment by the gas-air paths of the coal-fired machines of thermal power plants, dis.cand. (2015)

7. L.R. Yablonick, Noise protection structures of turbine and boiler equipment: theory and calculation, dis.cand. (2004)

8. I.B. Zayats, Noise reduction in the operation of gas distribution stations of main gas pipelines, dis.cand. (2015)

9. V.B. Tupov, Noise Reduction from Power Equipment, 232 (2005)

10. A.A. Igolkin, Development of a method and means of reducing aerodynamic noise in pneumatic and gas transport systems (2014)

11. E.Ya. Yudin, L.A. Borisov, I.V. Gorenshtein, Noise Abatement at Work, 400 (1985)

12. N.I. Ivanov, Engineering acoustics. Theory and practice of noise control, 4, 432 (2015)

13. M.V. Vasineva, Design solutions to protect the public from noise, Scientific Journal KUBSAU, 109(05) (2015)

14. A.V. Kuzmitskiy, A.V. Nikiforov, A.V. Ivanov, Evaluation of the acoustic impact in the residential area and in the premises using the software complex ARM "Acoustics" 3D, Biosphere compatibility: man, region, technology, 3 (2004)

15. O.A. Gorbunova, G.I. Pavlov, Development of an experimental-theoretical model of the noise field of the power equipment of thermal power plants, News of higher educational institutions. Problems of energy: Scientific, technical and industrial journal, 5/6, 84-92 (1998) 